\title{
CONTACT DAMAGE IN ARTIFICIALLY AGED 3Y-TZP
}

\author{
Z de Armas Sancho, A Mestra, E Jiménez-Piqué and M Anglada \\ Centro de Integridad Estructural y Fiabilidad de los Materiales, CIEFMA \\ Dpto de Ciencia de los Materiales e Ingeniería Metalúrgica \\ Universitat Politècnica de Catalunya, UPC \\ Av. Diagonal 647, 08028 Barcelona, Spain \\ zamirfim@yahoo.es
}

\begin{abstract}
Tetragonal zirconia polycrystals doped with $3 \%$ mol of yttria (3Y-TZP) are increasingly employed as implants in the human body, because they are biocompatible and have relatively high strength and fracture toughness. These properties are related to the small submicrometer grain size and to the operation of a toughening mechanism around cracks which is referred to as phase transformation toughening. Another important feature of 3Y-TZP is the phenomenon of low temperature degradation (LTD) that takes place in contact with water at temperatures in an interval around $250^{\circ} \mathrm{C}$. Water activates $\mathrm{t}-\mathrm{m}$ transformation at the surface and progresses inside the body producing a $\mathrm{t}-\mathrm{m}$ surface transformed layer. As a result, roughness and microcracking are produced in the surface. In a first stage, for short times of ageing, this layer is responsible for degradation of surface mechanical properties; at a later stage, after long degradation times, it induces a strong reduction in the bulk strength.

In this paper we present a study of the mechanical behaviour of hydrothermal degraded 3Y-TZP under monotonic and cyclic spherical indentation. The investigation is focused mainly on analyzing the mechanical response of the material under spherical indentation in terms of the degraded layer. Plastic deformation and cracking induced by spherical contact loading are studied under increasing loads in hydrothermally degraded and in as sintered specimens. The contact hardness and elastic modulus of the degraded layer are extracted from nanoindentation using continuous stiffness measurements. It is found that permanent damage induced in degraded specimens is always larger than in as sintered specimens. Critical loads to induce ring cracking in degraded specimens are lower than in as sintered specimens.
\end{abstract}

KEY WORDS: Contact Fatigue, Strength Degradation, Zirconia.

\section{INTRODUCTION}

Tetragonal zirconia polycrystals doped with $3 \%$ mol of yttria (3Y-TZP) are increasingly employed as implants in the human body because of their biocompatibility and relatively high strength and fracture toughness (1000 $\mathrm{MPa}, 5 \mathrm{MPam}^{1 / 2}$ ) [1]. The reason for these relatively high mechanical properties is a small grain size and a phase transformation toughening mechanism based on the stress-assisted tetragonal to monoclinic (t-m) transformation around the crack tip, which is accompanied by an increase in volume of about $4 \%$ [2]. This transformation leads to the development of localis ed compressive stresses around the crack flanks inducing in this way a drop in the crack tip stress intensity factor.

Another important feature of 3Y-TZP is the phenomenon referred to as low temperature degradation (LTD) that takes place when the material is exposed to water. Water activates the $\mathrm{t}-\mathrm{m}$ transformation at the surface, which progresses in to the bulk producing a monoclinic surface layer [3]. As a result, the surface roughness is increased and micro-cracking appears inside this layer. Initially, only the mechanical properties of the surface are degraded, with lower hardness and elastic modulus than the interior [4]. But, at later stages, a strong reduction in the bulk strength is also observed. LTD is easily detected after a few hours of degradation at temperatures around $250 \mathrm{C}$, but it has been also observed on femoral heads of 3Y-TZP of hip-joint replacements several years after implantation [5].

In the present work, we have studied damage under spherical contact fatigue in 3Y-TZP previously degraded by exposure to LTD for different periods of time. The results are compared with the behaviour of the nondegraded as sintered material.

Hertzian indentation is a method very extended for studying contact mechanics in brittle materials [6]. This methodology allows evaluating the damage and deformation produced by the contact of a sphere with a solid surface. One advantage is that it allows observing the evolution of the damage mechanisms through a progressive transition from the initial elasticity regime until the development of full plastic regime. This is not possible with an indent with a sharp tip since plastic deformation takes place from the beginning of the contact with the surface. In addition, there are analytical expressions for the elastic problem of a solid under Hertzian contact loading.

In ceramic materials, the contact of a sphere with the surface induces two main types of damage [7]. One occurs in low fracture toughness ceramics and is the 
appearance of a circular crack near the radius of contact of the sphere with the surface, which eventually extends into the bulk in the form of a cone. This type of damage was reported by Hertz as early as in 1881 (cone cracking).

Another type of damage may occur in tough ceramics and consists in the appearance of a "cuasi-plastic" damage zone below the contact area which is characterized by the formation of microcracks [8].

Numerous studies are reported about contact fatigue in ceramics [9-11]. In the present work, the fatigue by spherical contact in $3 \mathrm{Y}-\mathrm{TZP}$ has been studied for several times of LTD. Due to the loads and to the indenter radius, the damage zone studied includes both the transformed layer formed during degradation, as well as the non-affected tetragonal bulk.

\section{MATERIALS AND METHODS}

The starting material was commercial TZ-3YSB-E powder (Tosoh, Japan), with an average crystallite size of $27 \mathrm{~nm}$. About $40 \mathrm{~g}$ of powder was placed in a cylindrical polymeric mould in an ultrasonic bath for 15 minutes in order to homogenize the powder. Then, the closed mould was cold isostatically press under a $200 \mathrm{MPa}$ applied pressure during 3 minutes. The produced green bodies were sintered at $1450{ }^{\circ} \mathrm{C}$ for 2 hours with heating and cooling ramps of $3^{\circ} \mathrm{C} / \mathrm{min}$.

The cylinders were cut into $2 \mathrm{~mm}$ thick discs, which were ground with a diamond disc of $68 \mu \mathrm{m}$ average particle size, and finally they were polished up to colloidal silica finish $(30 \mathrm{~nm})$ in order to have mirror-like appearance. The density was measured by Archimedes method and it was found to be $6.01 \mathrm{~g} / \mathrm{cm}^{3}$ which is $99 \%$ of the theoretical density.

Hydrothermal ageing was performed in an autoclave maintaining a steam atmosphere at a temperature of 131 ${ }^{\circ} \mathrm{C}$ and at pressure of 2 bars for different times (10, 30 and 60 hours). The specimens degraded for these times will be referred to as D10, D30 and D60 respectively, while the non-degraded as sintered specimens will be referred to as "AS".

The microstructure and damage were observed by optical microscopy, confocal laser microscopy and scanning electron microscopy (SEM). The monoclinic phase content was analysed by micro-Raman spectroscopy and atomic force microscopy (AFM).

\subsection{Monotonic Spherical Indentation}

Several types of monotonic tests were carried out. Contact hardness and elastic modulus were determined by nanoindentation with a Berkovich tip up to $2 \mu \mathrm{m}$ depth. The Berkovich tip was calibrated against a standard fused silica. Nanoindentation tests were performed with a MTS Nanoindenter XP with a continuous stiffness measurement (CSM) module, which allows continuous measurement of contact stiffness (S) and load (P) as a function of penetration depth (h). Indentations were made to a maximum load of $500 \mathrm{mN}$ to a maximum penetration depth of about $1600 \mathrm{~nm}$, and under a constant deformation velocity of $0.05 \mathrm{~s}^{-1}$. Nine indentations were made as $3 \times 3$ matrices on each material and the results were presented with standard deviation. The values of hardness $(\mathrm{H})$ and Young's modulus (E) were calculated as a function of penetration depth using the method of Oliver and Pharr [12]. In this method, the contact depth is estimated from the load-displacement data using:

$h_{c}=h_{\max }-\varepsilon \frac{P_{\max }}{S}$

Where, $P_{\max }$ is the peak indentation load and e is a constant which depends on the indenter geometry [13] and $\mathrm{S}$ is the contact stiffness. Empirical studies have shown that $\mathrm{e}^{\sim} 0.75$ for a Berkovich indenter. From the measurements of load and displacement data, the projected contact area, $A$, of the indentation impression is estimated by evaluating indenter shape function at the contact depth, $h_{\mathrm{c}}$; that is $A=f\left(h_{\mathrm{c}}\right)$. The shape function, $f(d)$, relates the cross-sectional area of the indenter to the distance, $d$, from its tip.

Once the contact area is determined from the load displacement data, the hardness, $H$, and effective elastic modulus, $E_{\text {eff }}$, were calculated as follows.

$$
\begin{aligned}
& H=\frac{P_{\max }}{A} \\
& E_{\text {eff }}=\frac{1}{\beta} \frac{\sqrt{\pi}}{2} \frac{S}{\sqrt{A}}
\end{aligned}
$$

Where $\beta$ is equal to 1.034 for a Berkovich tip. This technique provides data with continuous measurement of the hardness and elastic modulus as a function of depth.

Nanoindentation with a spherical tip of radius $\mathrm{R}=25.75 \mu \mathrm{m}$ was also used to record the elastic and plastic regimes of deformation. For a spherical indenter of radius $R$, in the elastic regime there is a linear relation between the average contact pressure $p_{0}$ and the indentation strain, $a / R[6]$ :

$$
p_{0}=\left(\frac{3 E}{4 \pi k}\right) \cdot \frac{a}{R}
$$

where $p_{0}$ is defined as a function of the applied load $F$ and the contact radius $a$ :

$$
p_{0}=\frac{F}{\pi a^{2}}
$$


and $k$ is a constant which depends on the elastic properties of the indenter and the material:

$$
k=\frac{9 E}{16}\left(\frac{1-\mathrm{v}^{2}}{E}+\frac{1-\mathrm{v}^{\prime 2}}{E^{\prime}}\right)
$$

The contact radius is related to the depth of the circle of contact by:

$a \approx \sqrt{2 R h_{c}}$

Here, E, $v$ and $E^{\prime}, v^{\prime}$ are elastic modulus and Poisson's ratio of the material and indenter, respectively.

The average indentation pressure was measured against $\mathrm{a} / \mathrm{R}$ and according to equation (4) in the elastic regime and a straight line should result. At higher pressures plastic deformation takes place, but still it is interesting to plot $\mathrm{p}_{0}$ against $\mathrm{a} / \mathrm{R}$ since by the emp irical Meyer's law it can be demonstrated that the curve obtained is independent of the radius of the sphere. Macroscopic tests with a sphere of larger radius were focused on obtaining the indentation stress - strain curves and the critical load for damage appearance. The tests were performed in a universal testing machine (Instron Model 8511), using cemented tungsten carbide spheres of radius $\mathrm{R}=1.25 \mathrm{~mm}$, in a range of loads between 500 and $3000 \mathrm{~N}$. The load was increased in steps of $100 \mathrm{~N}$. It was applied at a speed of $20 \mathrm{~N} / \mathrm{s}$ using a $50 \mathrm{~N}$ preload in laboratory air environment and with a relative humidity of $20-45 \%$. For each degradation condition, three samples were tested.

After unloading, each specimen was examined in the optical microscope to detect permanent deformation and surface cracks. If damage was detected, further closer observation was performed with a confocal laser microscope. In the monotonic contact tests, the criterion used to define the critical load for cracking was the appearance of a well developed ring crack around the residual imprint. When such crack was detected, the specimen was cut into two halves perpendicular to the surface and carefully polished in order to reveal any progression of the surface ring crack into the bulk to form a cone crack.

\subsection{Cyclic loading spherical indentation tests}

Fatigue tests were performed under the same conditions as in monotonic loading for a range of cycles between 10 and $10^{6}$. All fatigue tests were performed with a sinusoidal waveform of $15 \mathrm{~Hz}$ and $\mathrm{R}=0.1$. According to the critical loads obtained for cracking in the monotonic tests, three load levels were chosen for fatigue characterization $(500,750$ and $1000 \mathrm{~N})$.

\section{RESULTS AND DISCUSSION}

\subsection{Change in microstructure by degradation}

The effect of degradation is to induce monoclinic phase in a surface layer. Although its concentration is not constant along the depth direction, as a first approximation, this layer will be considered as it had constant monoclinic concentration, that is, it shall be treated as a "coating" with well defined mechanical properties.

After long degradation times (more than 10 hours), the concentration of monoclinic phase is practically constant into the depth direction until there is a relatively sharp drop. Table 2 gives the main characteristics of the degraded layers.

Table 1. Berkovich nanoindentation elastic modulus and hardness of AS and degraded layers.

\begin{tabular}{|c|c|c|c|}
\hline & $\begin{array}{c}\text { Thickness of } \\
\text { degraded layer } \\
(\mu \mathrm{m})\end{array}$ & $\begin{array}{c}\text { Elastic } \\
\text { Modulus } \\
(\mathrm{GPa})\end{array}$ & $\begin{array}{c}\text { Hardness } \\
(\mathrm{GPa})\end{array}$ \\
\hline AS & - & 220 & 16 \\
\hline D10 & $\sim 2$ & - & 15 \\
\hline D30 & 6.3 & - & 13 \\
\hline D60 & 12 & 170 & 11 \\
\hline
\end{tabular}

Because of the existence of micro-cracks in this degraded layer [5], its elastic modulus is smaller than for the inner bulk material. That is, being the degradation layer more compliant, it will take up more deformation than AS.

\subsection{Monotonic Spherical Contact Loading}

Figure 1 shows the penetration curves up to a load of $600 \mathrm{mN}$ obtained from instrumented indentation with a spherical tip.

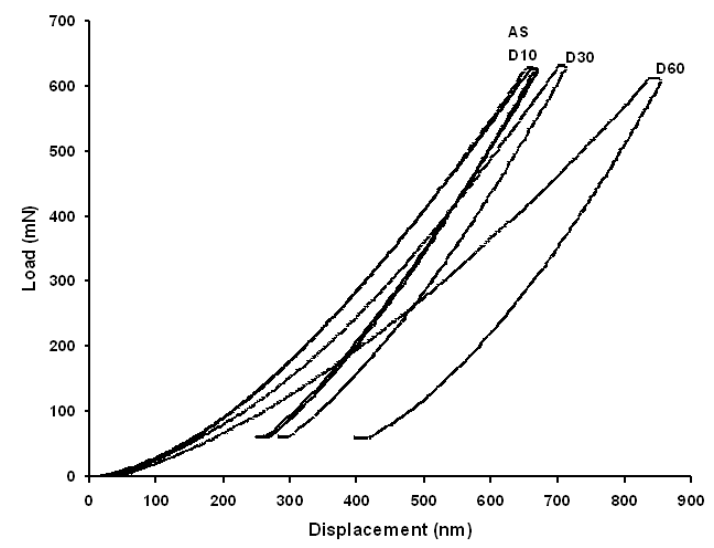

Figure 1: Load vs. displacement, instrumented nanoindentation

It can be appreciated that for specimens degraded for more than 10 hours the total and inelastic displacements are larger. This is in line with a thicker degraded layer produced during longer degradation times as well as with the fact the degraded layer is more compliant and has a lower strength than the "substrate" (AS material). 


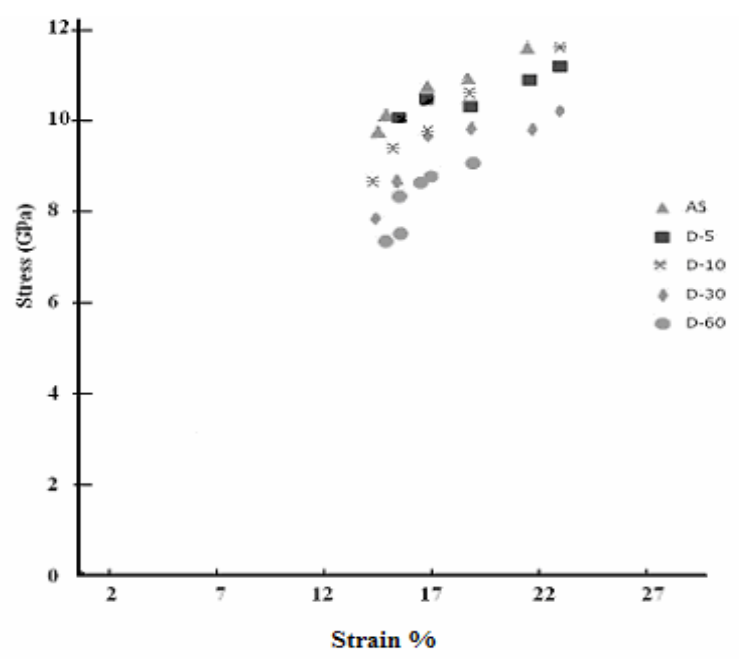

Figure 2. Mean pressure versus strain in spherical indentation tests.

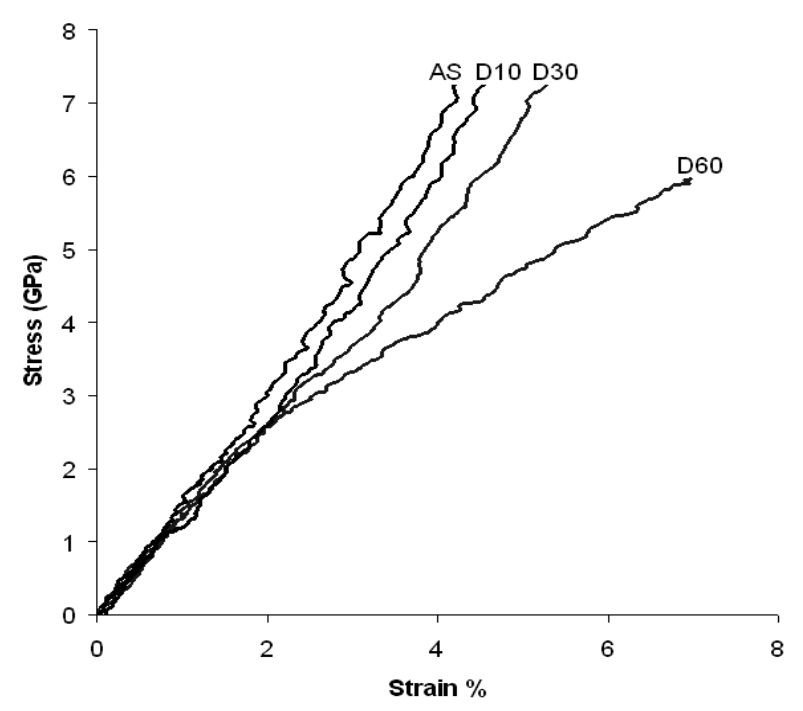

Figure 3. Stress vs. strain in nanoindentation test

Figure 2 shows the curves of mean pressure $p_{0}$ against indentation strain obtained by using the ball of $1.2 \mathrm{~mm}$ diameter in the specimens in the servo-hydraulic testing machine at high loads. It can be appreciated that the stress necessary to induce a given plastic strain in degraded specimens is smaller than in AS.

In degraded specimens, the effective elastic modulus of degraded layer + substrate measured by nanoindentation depends on the time of degradation and the depth of penetration. When the nanoindentation main elastic contribution comes from the degraded layer, the value of the elastic modulus for the degraded layer was found to be equal to about $170 \mathrm{GPa}$. However, it is obvious that in our macroscopic spherical indentation tests the elastic contribution of the "substrate" is very large because of the small thickness of the degraded layer in comparison with the contact radius. Because only residual deformation was measured in our macroscopic experiments, all experimental points are in the plastic regime. In order to measure the elastic and transition regime we use instrumented indentation with the contact area measured from the contact stiffness (see figure 3 ). Here it is very clear the proportionality between pressure and strain is lost at smaller pressures for the degraded specimens.

The elastic modulus for AS calculated from figure 3 (230 $\mathrm{GPa}$ ), is close to the value of $220 \mathrm{GPa}$ reported by Berkovich nanoindentation. The elastic modulus measured for D60 was $182 \mathrm{GPa}$, that is, lower than for AS. It is clear from the results shown in figure 3 that D60 exhibits a deviation from the elastic behaviour earlier than the rest of the samples.

The first damage observed is the residual deformation circular imprint and subsequently the formation of circular cracks around the first. As the load level was increased, the circular crack progresses towards the interior of the material in the form of a cone crack, as can be seen in figure 4 .

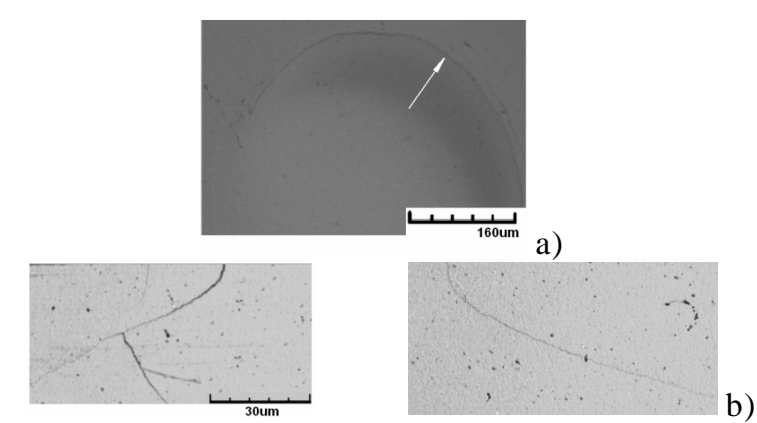

Figure 4. a) ring cracks on the top surface; b) cone cracks in the cross section.

The critical load $P_{c}$ and the critical pressure $p_{o c}$ for the appearance of the circular cracks in each one of the degradation stages of the material are given in table 2 . These values correspond to the minimum monotonic load able to produce a complete circular crack around the residual imprint.

Table 2. Critical loads for the appearance of circular cracks in monotonic and cyclic contact loading

\begin{tabular}{|c|c|c|c|}
\hline Sample & $\begin{array}{c}\mathrm{P}_{\mathrm{c}} \text { (mont) } \\
(\mathrm{N})\end{array}$ & $\begin{array}{c}\mathrm{p}_{0 \mathrm{c}} \\
(\mathrm{GPa})\end{array}$ & $\begin{array}{c}\text { Pfc } \\
(\mathrm{cyclic}) \\
(\mathrm{N})\end{array}$ \\
\hline D60 & 1500 & 8.88 & 750 \\
\hline D30 & 1800 & 9.95 & 900 \\
\hline D10 & 2300 & 10.6 & 1150 \\
\hline AS & 3000 & 12.2 & 1500 \\
\hline
\end{tabular}

\subsection{Cyclic Spherical Contact Damage}

The results of the contact fatigue tests for the three levels of maximum load tested are represented in figure 4 where it can be seen the number of cycles imposed at 
each level together with information about the appearance of damage in form of a circular crack. The figure reveals that the material is always sensitive to fatigue. Clearly the critical amplitude for crack damage appears at loads lower than Pc (load for cracking under

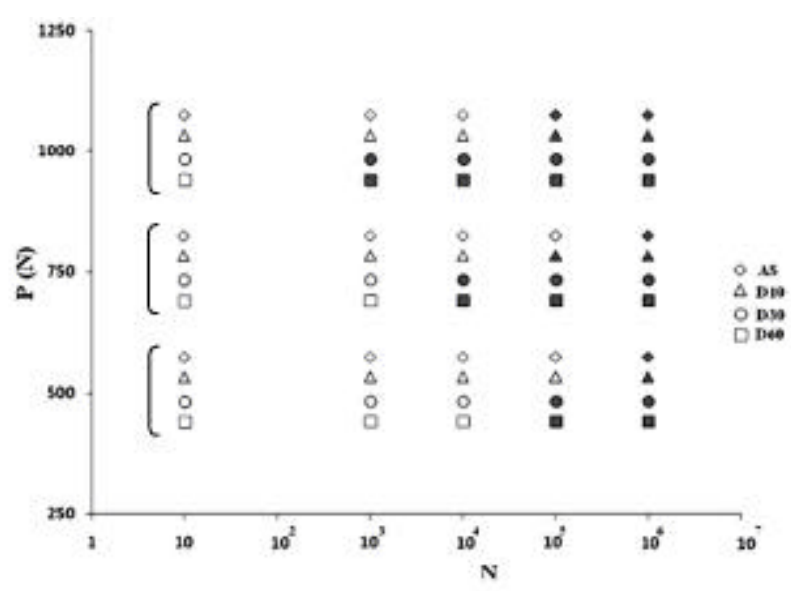

Figure 4. Load versus numbers of cycles. No critical damage is shown by open symbols whereas the presence of circular cracks is shown by closed symbols.

monotonic loading), and it diminishes as we increase the number of cycles. It can be also observed than the number of cycles needed for the appearance of damage depends on the duration of hydrothermal ageing. The degraded materials D30 and D60 offer a much lower resistance to contact loading. The non-degraded material does not reveal damage until a number of cycles of at least two orders of magnitude higher than for the degraded specimens.

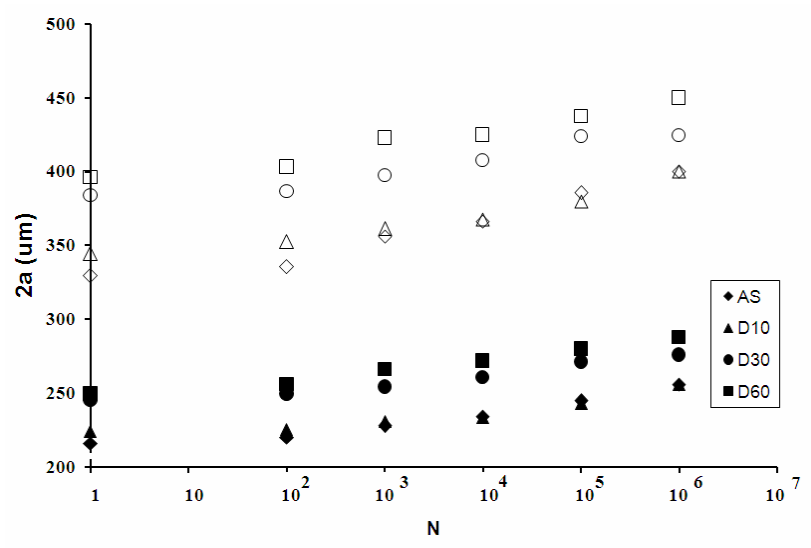

Figure 5. Residual spherical imprint diameter vs. number of cycles for two load levels: $1000 \mathrm{~N}$ (open symbols) and $500 \mathrm{~N}$ (closed symbols).

In figure 5, the diameter of the residual plastic imprint is plotted in terms of the number of cycles for the different cyclic loads and specimen conditions. For a given load level, the diameter of the residual imprint increases with the number of cycles. In addition, longer degradation times induce larger residual deformation for a fixed number of cycles
Figure 6 displays the appearance of the residual imprint on the surface in terms of number of cycles and time of degradation. After the first $10^{4}$ cycles, the residual imprint is clearly developed for all conditions. For larger number of cycles, two regions are observed: one rough annular region and the centre which is smoother.

The central part of the imprint is subjected to high normal pressure while just outside the contact radius is where there is the higher tensile stress and where the normal stress becomes zero. For AS at the surface near the contact radius, the maximum Hertz tensile stress is much lower than the yield point as well as for inducing either transformation or cracking. However, in the aged condition, the yield strength of the degraded layer is much lower than for the "substrate". Here from figure 3 it can be noticed that yielding for D60 takes place at stresses which are about $1 / 3$ of the yield strength of AS. This has been also shown by Adrian e al [13] who showed that the system substrate+degraded layer could have a bend strength as low as $25 \%$ after ( 150 hours of ageing). However these stresses are higher than the maximum tensile elastic stress near the contact radius.

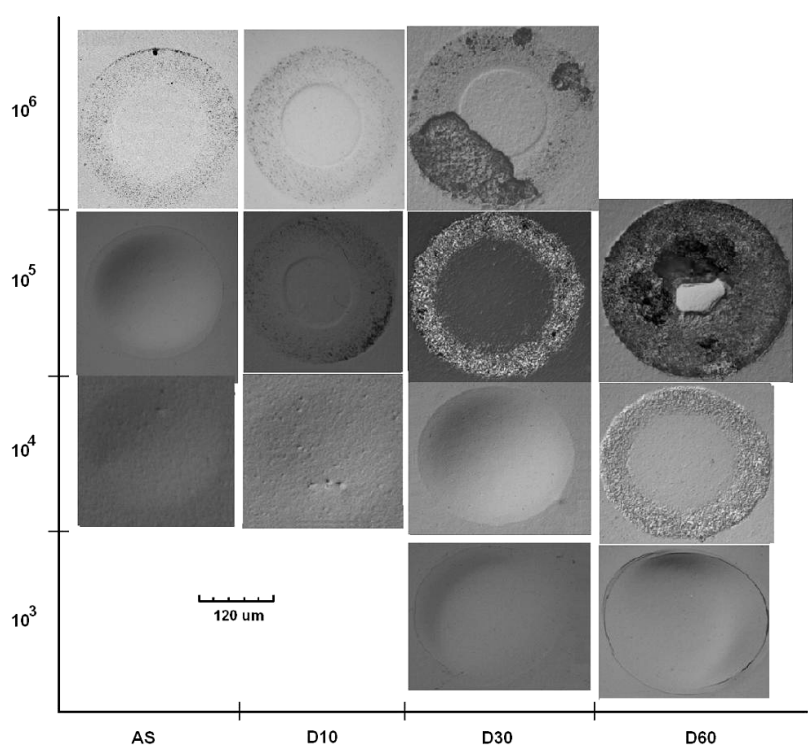

Figure 6. Evolution of the residual spherical imprint radius and damage with increasing number of cycles and time of degradation for a load amplitude of $500 \mathrm{~N}$.

In the degraded layer there are microcracks which can be the nucleus for the generation of the ring crack. This is the reason for the earlier appearance of ring cracks in degraded specimens. This effect is stronger for specimens degraded for longer times. In this case not only there are many nuclei on the surface, but also in the depth direction, so that the extension of the ring crack into the depth will be easier.

For degraded specimens the first damage mechanism that takes place after the generation of the circular crack is the pull out of grains in an annular region close to the contact radius. Later there is superficial chipping in this region. Finally, a mechanism of fracture by layers appears that progresses inside the material. 
For AS, the mechanisms of pull - out and micro-chipping take place for a number of cycles higher that $10^{5}$ cycles, as it is observed in figure 7 . In this material the susceptibility to damage will be marked by the transformation $\mathrm{t}-\mathrm{m}$ that takes place during the process of cyclic indentation, since there is not initial microcracked region.

Micro-Raman spectroscopy analysis was carried out on AS specimens after $10^{5}$ and $10^{6}$ cycles of loading at a maximum load of $500 \mathrm{~N}$ (see, Table 3). Outside the contact zone no monoclinic phase was detected.
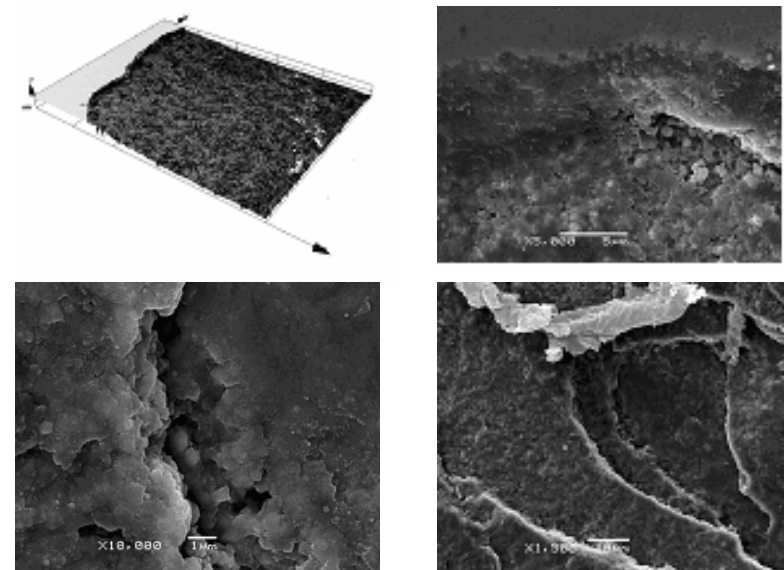

Figure 7. Damage for D30: a) Circular crack border. b) Pull out in the surface near de circular crack. c) Detail of circular crack; d) Final failure.

Table 3. Micro-Raman analysis on imprint of AS after contact fatigue with a maximum load of $500 \mathrm{~N}$.

\begin{tabular}{|c|c|c|}
\hline Cycles & $\begin{array}{c}\text { Zones } \\
\text { (residual imprint) }\end{array}$ & $\begin{array}{c}\text { Monoclinic phase } \\
\text { (volume \%) }\end{array}$ \\
\hline \multirow{2}{*}{$10^{5}$} & Center & 7 \\
\cline { 2 - 3 } & Border & 12 \\
\hline \multirow{2}{*}{$10^{6}$} & Centre & 13 \\
\cline { 2 - 3 } & Border & 20 \\
\hline
\end{tabular}

\section{CONCLUSIONS}

The behaviour of 3Y-TZP under spherical indentation and contact fatigue has been studied in specimens subjected to LTD for different times of up to 60 hours. The main conclusions are:

1. The effect of degradation is clearly revealed by instrumented indentation and the degraded layer has lower hardness and elastic modulus than the substrate reaching yield strengths of about $25 \%$ after 60 hours of degradation.

2. The permanent deformation induced in degraded specimens is always larger than in the as sintered material.

3. The critical loads to induce ring cracking in degraded material are lower than in the as sintered material.

4. The damage induced by contact fatigue is larger in the specimens degraded for longer times, so that the degraded layer plays an important role in the failure related contact response of $3 \mathrm{Y}-\mathrm{TZP}$.

\section{ACKNOWLEDGEMENTS}

Financial support from Spanish "Ministerio de Ciencia e Innovación" under the project MAT 2008-03398 is greatly acknowledged. Z. de Armas acknowledges a scholarship from the Alßan Programme of the EU.

\section{REFERENCES}

[1] Helmer J D, Driskell T D "Research on bioceramics" Symposium on Used of Ceramics as surgical Implants Clemson, South Carolina: Clemson University (1969).

[2] Poter D L, Heuer A H. "Mechanics of toughening partially stabilized zirconia (PSZ)” J. Am. Ceram Soc 60:183-184 (1977)

[3] Kobayashi K Kuwajima H, and Masaki T "Phase change and mechanical properties of $\mathrm{ZrO} 2-\mathrm{Y} 2 \mathrm{O} 3$ solid electrolyte after ageing" Solid State Ion 3(4) 489-493 (1980)

[4] Gaillard Y, Jiménez E P, Soldera F, Mucklich F, Anglada M "Quantification of hydrothermal degradation in zirconia by nanoindentation" Acta Materialia Volume 56 Issue 16 Pages 4206-4216

[5] J. Chevalier, L. Gremillard and S Deville, "Low temperature degradation of Zirconia and implications for Biomedical Implants". Annu. Rev. Mater. Res. 37, 1-32 (2007)

[6] Lawn, B.R. "Indentation of ceramics with spheres: a century after Hertz.”J. Am. Ceram. Soc. 81.8 (1998): 1977-1994.

[7] Rhee, Y.-W., Kim, H.-W., Deng, Y., and Lawn, B.R. "Brittle fracture versus quasi plasticity in ceramics: a simple predictive index.” J. Am. Ceram. Soc. 48.3 (2001): 561-565.

[8] Lawn, B.R., Padture, N.P., Cai, H., and Giberteau, F. "Making ceramics "ductile"." Science 263 (1994): 1114-1116.

[9] Kim, D.K., Jung, Y.-G., Peterson, I.M., and Lawn, B.R. "Cyclic fatigue of intrinsically brittle ceramics in contact with spheres." Acta mater. 47.18 (1999): 4711-4725.

[10] Lee, K.S., Jung, Y.-G., Peterson, I.M., Lawn, B.R., Kim, D.K., and Lee, S.K. "Model for cyclic fatigue of quasi-plastic ceramics in contact with spheres. ”J. Am. Ceram. Soc. 83.9 (2000): 2255-2262.

[11] J. Zhou, J. Mah, P. Shrotriya, C, mercer, W. O. Soboyejo. "Contact damage in an yttria stabilized zirconia: Implications for biomedical applications”. J. Mater Sci: Mater Med (2007) 18:71 - 78

[12] W. C. Oliver and G. M. Pharr: "Measurement of hardness and elastic modulus by instrumented indentation: Advances in understanding and refinements to methodology". J. Mater. Res. 19, 3 (2004).

[13] A. Feder, M. Anglada. "Low-Temperature Ageing Degradation of $2.5 \quad Y$-TZP HeatTreated at $1650^{\circ} \mathrm{C}$," J. Eur. Ceram. Soc., 25, 3117-24 